\title{
Advanced Mobile Security System Operated by Bioelectrical Sensor
}

\author{
Kalanyu Zintus-art ${ }^{1}$, Duk Shin ${ }^{2 *}$, Natsue Yoshimura ${ }^{2}$, Hiroyuki Kambara ${ }^{2}$ and \\ Yasuharu Koike ${ }^{1,2}$ \\ ${ }^{1}$ Department of Information Processing, Tokyo Institute of Technology, Japan \\ ${ }^{2}$ Precision and Intelligence Laboratory, Tokyo Institute of Technology, Japan \\ \{kalanyuz, shinduk, yoshimura, hkambara, koike\}@cns.pi.titech.ac.jp
}

\begin{abstract}
This article describes a novel type of an advanced mobile security system, a myokinetic password, using surface electromyographic (EMG) signals. We proposed an advanced security system that can increase password strength without modifying password length or increasing alphabet character variation. We present an implementation of an intuitive password system that combines muscle activation to enhance password complexity.

The muscle activation levels from EMG signals are used to split each individual code composition into multiple states, thereby increasing possible password combinations. We proposed a centroid-based clustering algorithm to cluster a feature into groups according to the muscle activation. We also verified the possibility of expanding the technique to support up to three muscle activation levels in the experiment. By utilizing the proposed method, the number of possible input key combination is increased from 256 to 810,000 with three muscle levels.
\end{abstract}

Keywords: electromyogram, security, password, muscles, bioelectrical sensor

\section{Introduction}

Numerical password identifications have long been applied in various technologies. Electronic devices such as automated teller machines (ATMs), security doors, and mobile devices adapt the system due to the design aspects that allow easily memorable passwords, high verification accuracy, and fast input method. With these conveniences, however, fatal disadvantages exist. Numerical passwords in such system have small combinations and can be easily cracked using simple algorithms $[1,2]$. Ones password characters are leaked to third parties, no immediate countermeasures that prevent or mitigate damages, such as prolonging the safety period before successful exploitation, are provided. Thus, numerous researches are conducted in order to create a more robust and secure system.

Apart from increasing the complexity of password characters, different types of password and input method are proposed to provide robust security. For example, Rass, et al., (2010) presented a detailed study of using Face-Recognition based authentication [3]. Also, the use of biometrical data for identity identification has received tremendous amount of attentions during the past decades. Tan, et al., (2002) developed an approach to match fingerprints using Genetic Algorithms, which provides improved performance [4]. Hill, et al., (2002) described the possibility of using retinal images as identification method and its disadvantages [5]. However, these systems are either not user-friendly or too expensive to be implemented in large scales. To look for a source of biometrical data that will not require drastic changes in

${ }^{*}$ Corresponding Author 
input method, muscles are worth mentioning because they correlates with the way we provide input to a system.

Human muscles are very complex. Even though body posture remains unchanged, humans can control their muscle activities in a way that reflects force or resistance. These kinds of activities often result in different levels of electromyography (EMG) recordings that can be distinguished from each other [6, 7]. However, biosignals such as EMG are very sensitive and subject-specific. Gender, age, and various body conditions can effect the readings obtained from a user. Therefore, the signals need to be normalized and observed before meaningful patterns can be analyzed.

In this study, we developed a 4-digits numerical password system on an iPad with an EMG sensor attached to the user's forearm. The EMG signal from the sensor is transferred to a PC and wirelessly synchronized with touch events on the iPad where the a combination consisting of numerical characters and muscle activity levels are used as the system's input. The password authentication process is done using a within-user muscle activity recognition algorithm constructed based on training data. The algorithm is then validated on the same set of users to assess repeatability and usability.

This paper is organized as follows: Section 2 introduces the system along with its implementation and the development tools. Experiment details and algorithms are also included in Section 2. Experimental results are described in Section 3. Section 4 discusses the potential of the constructed system and Section 5 concludes this paper.

\section{Methods}

\subsection{EMG - Digit Pass Code Security System}

We used a desktop EMG system, BagnoliTM 4, which is a complete EMG sensing solution developed by Delsys Inc. The EMG Systems has been deployed in various researches, such as motor control units analysis [8], presymptomatic indication [9], speech recognition [10], sport performance assessment [11], prosthetic limbs design [12], and computer peripheral design [13]. The system consists of arrays of sensors and that can be placed on various muscles to detect electrical potential generated by muscles, allowing applications on fields such as medical and Human-Computer Interaction possible.

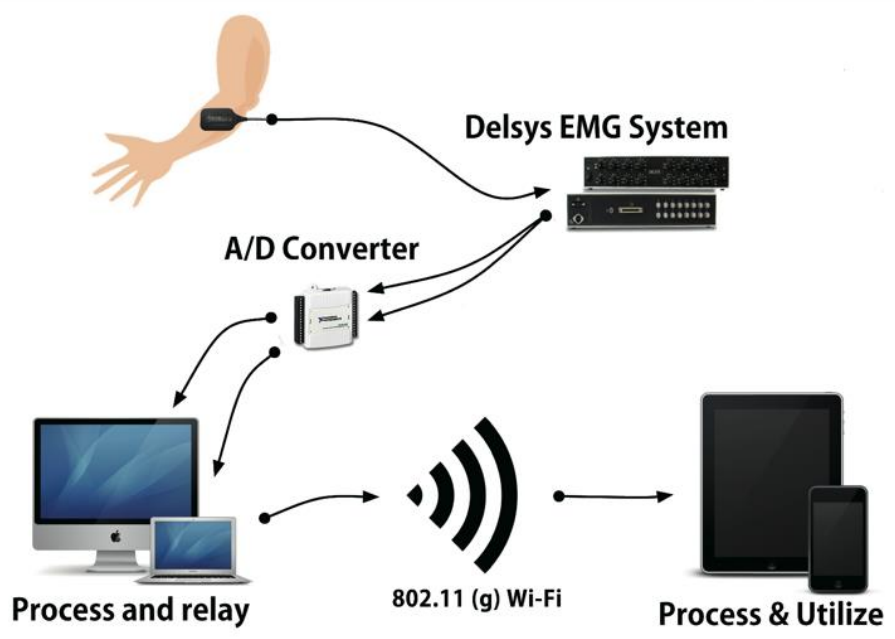

Figure 1. Proposed System Architecture 
The security system consists of two primary parts: The 4 digit passcode system was simulated on Apple's iPad and the data collection system built on Mac OS X using Apple's Mac OS X SDK. The touch events on the iPad are then synchronized with the data collection system through socket programming techniques. After a user completed his/her password input, the EMG data for the entire button pushes and the password characters are sent for authentication. Figure 1 shows the illustration of the proposed system.

\subsection{Procedure}

The users sat on a chair with the iPad. We recorded EMG signal of flexor carpi ulnaris using active surface electrodes in a bipolar configuration. After attaching EMG sensors, we performed EMG calibration prior to validation test because there are personal differences on EMG activities. Then, one user was asked to perform three types of experimental tasks for algorithm as follows:

- Task1: Button push with muscle co-contraction, 2 activation levels.

- Task2: Normal button push, 2 activation levels.

- Task3: Normal button push, 3 activation levels.

To validate the accuracy of the security system in regards of repeatability, five subjects were asked to test a 4-digits numerical password system where the subjects will have to repeat 10 password inputs into the system according to the predefined values without prior training.

\subsection{Preprocessing}

For preprocessing, the incoming signal is first normalized for real-time system with reference to the algorithm in Kalanyu, et al., [14] where initial calibration is required. For this system, each subject was asked not to activate any muscle activity for two seconds for the calibration to take effect. Here, the number of data needed for finding the maximum values is 2,000 . This corresponds to the $1 \mathrm{kHz}$ sampling rate with 16-bit resolution used in data collection. Also, mean is used instead of median due to the concern of high data variation resulting from the signal amplification rate applied. The modified algorithm is as follow:

$$
\frac{S(t)-}{\max _{i \in\{1,2, \ldots, 2000\}} C(i)}
$$

where $S$ is the signal in time $t$, and $\mu$ is the mean of the calibration data $C$. After normalization, EMG signals digitally rectified and passed through a second-order lowpass filter (cut-off frequency: $2.2 \mathrm{~Hz}$ ) [15-17]. Figure 2 shows a portion of EMG signals before and after preprocessing.

\subsection{Feature Extraction}

For each task, training datasets were collected from each subject by asking them to perform three sets of button pushes with all task-specific muscle activation levels. Then, segmentation technique is used to segment incoming data for feature extraction. Since in the first task the muscle is activated before a button is pushed, data for each button is segmented from the previous button push to the current but not more than 500 milliseconds. 
(a)

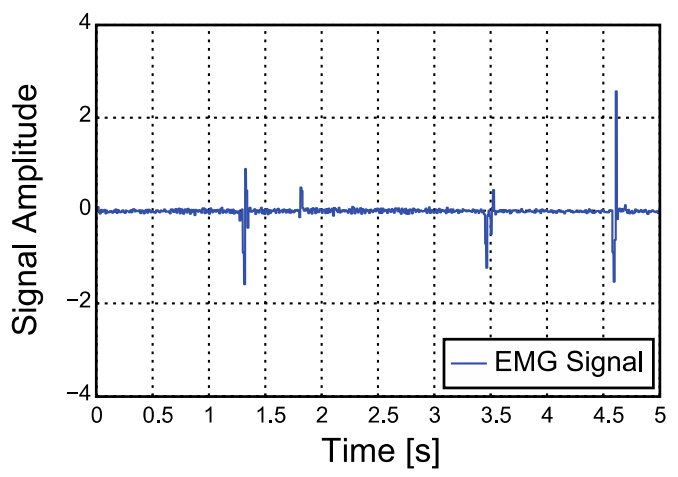

(b)

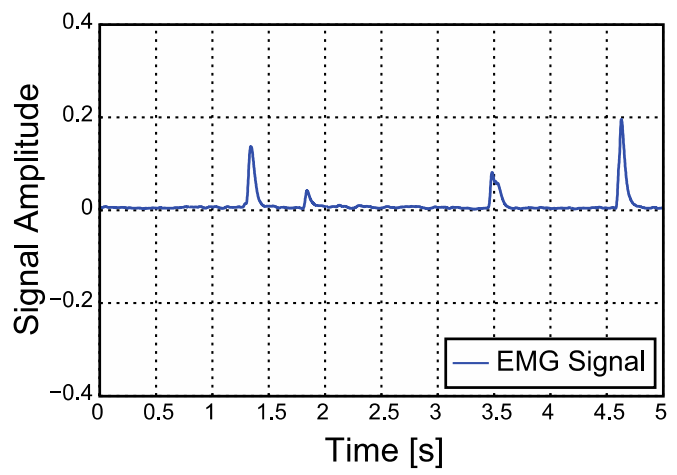

Figure 2. An Example of Preprocessed Signal: (a) a Portion of Signals before Processing and (b) the same Signals after Preprocessing

(a)

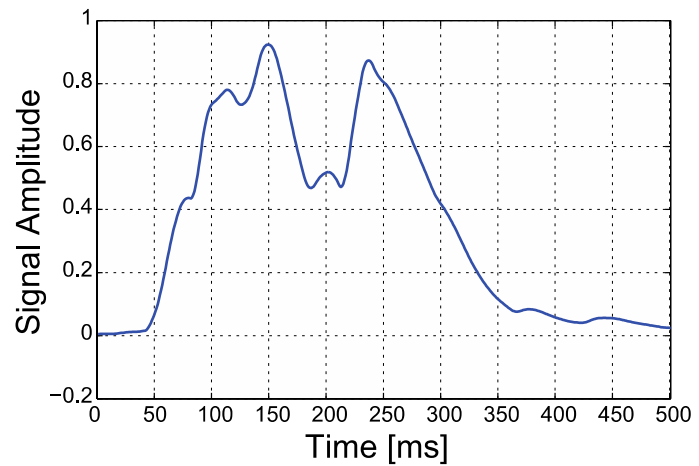

(b)

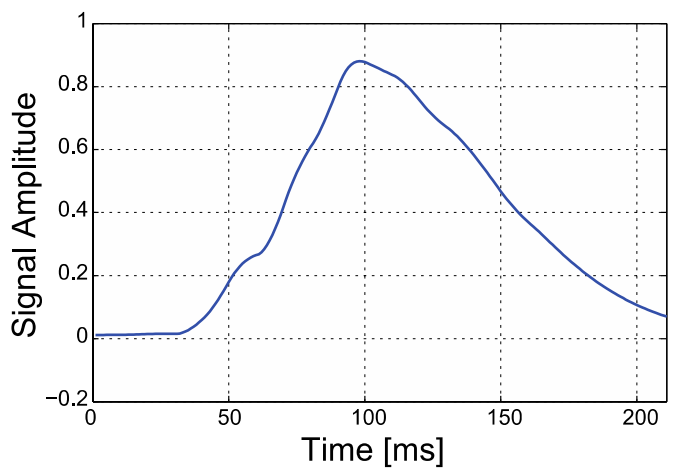

Figure 3. Example of Segmented Signals during Activation Period: (a) Signals Segmented before Button Push in task 1. (b) Signals Segmented During a Button Push in Task 2 and 3

This is different from the second and the third task where incoming data is segmented between 200 milliseconds before button push and the time the subject finger is released from the button. Figure 3 shows the data when segmented according to the task.For two muscle activation levels tasks, standard deviation is extracted from each segment and used as the classification feature. In case of three muscle activation levels, the time interval for each individual button push is also added as another feature. Figure 4 shows the characteristics of the features for two and three muscle activation levels tasks, respectively.

\subsection{Muscle Activity Level Recognition}

For each task, feature vectors calculated from each data segment are clustered into groups according to the muscle activation levels required. In order to cluster a feature vector of signal segment $i$, a simple but robust centroid-based clustering algorithm is proposed. The generalized form of the algorithm is as follows:

Step 1: The matrix containing feature vectors is normalized to eliminate the range difference between features using the following formula: 
(a)

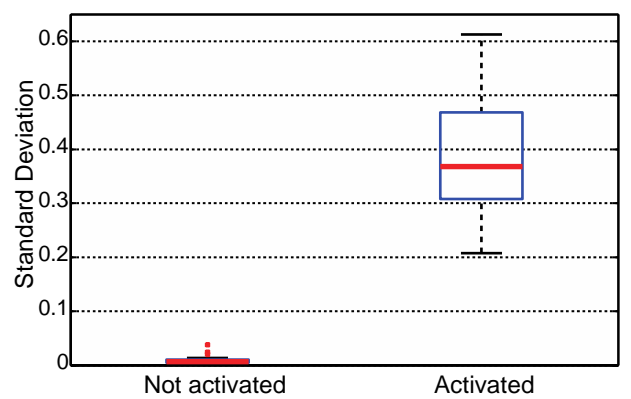

(c)

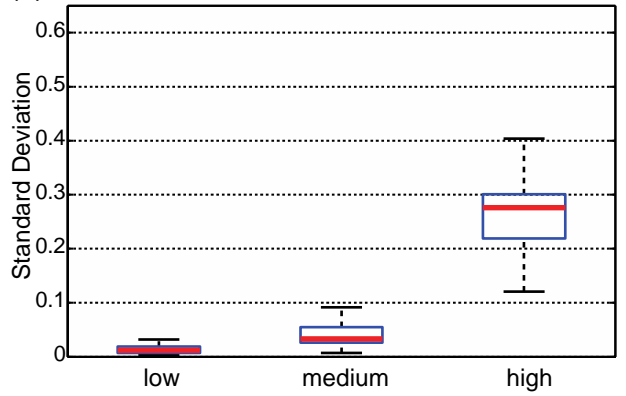

(b)

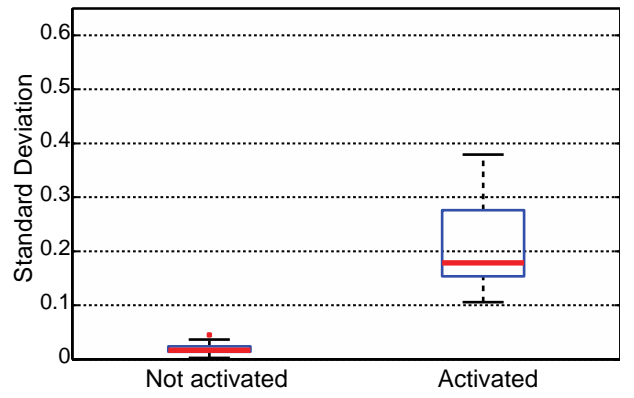

(d)

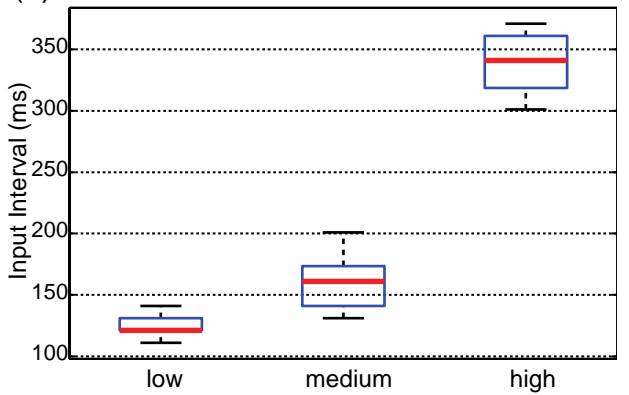

Figure 4. Box Plots Showing Feature's Characteristics of a Subject in Different Tasks: (a) Standard deviation for the first task. (b) Standard Deviation for the Second Task. (c) Standard Deviation for the Third Task. (d) Interaction Interval for the Third Task.

$$
\forall f \in F, \hat{X}_{i, f}=\frac{X_{i, f}-\min \left(T_{l, f}\right)}{\max \left(T_{h, f}\right)-\min \left(T_{l, f}\right)}
$$

where $\hat{X}$ is the normalized form of feature vector $X, f$ is feature number in feature space $F, i$ is the segment number, $T_{l}$ is the training data for "Not activated" and "Low", $T_{h}$ is the training data for "Activated" and "High", respectively.

Step 2: We define cluster centroids $C$ as follows:

$$
C_{v, f}=\frac{\sum_{i=1}^{N} \hat{T}_{l, f}}{N}
$$

here, $v$ stands for activation levels, $N$ is the total number of segments, and $\hat{T}$ is the normalized training data using Eq. 2.

Step 3: After cluster centroids are defined, distance between each muscle activation level $v$ and each feature vector $F$ is defined using Euclidean distance:

$$
D_{i, v}=\sqrt{\sum_{f=1}^{F}\left(\hat{X}_{i, f}-C_{v, f}\right)^{2}}
$$

Step 4: Finally, cluster label for the $i^{\text {th }}$ feature vector for two muscle activation level tasks is defined by:

$$
Y\left(\hat{X}_{i}\right)= \begin{cases}\text { Notactivated } & \text { if }, D_{i, l}<D_{i, h} \\ \text { Activated } & \text { if }, D_{i, h}<D_{i, l}\end{cases}
$$


For three muscle activation levels:

$$
Y\left(\hat{X}_{i}\right)= \begin{cases}\text { Low } & \text { if, }\left(D_{i, l}<D_{i . m}\right) \wedge\left(D_{i, l}<D_{i, h}\right) \\ \text { Medium } & \text { if },\left(D_{i, m}<D_{i, l}\right) \wedge\left(D_{i, m}<D_{i, h}\right) \\ \text { High } & \text { if },\left(D_{i, h}<D_{i . m}\right) \wedge\left(D_{i, h}<D_{i, l}\right)\end{cases}
$$

In this case, feature vector $\mathrm{F}$ for the first and the second task consist of $\{\sigma\}$ while the third task contains $\{\sigma, t\}$ where $\sigma$ denotes standard deviation of a signal segment and $\mathrm{t}$ denotes the time interval for the button push event of that segment. However, the resulting algorithm provides wide cluster regions for "Not activated" and "Low", which is not desired due to the stable characteristic of this level shown in Figure 4. Based on the hypothesis that the signal remains steady on the baseline with low variance during non-activation period, the following modification is applied during the computation of $D_{i, l}$ to reduce the region of "Not activated" level:

$$
D_{i, l}=\sqrt{\sum_{f=1}^{F}\left(\hat{X}_{i, f}-C_{l, f}\right)^{2}} \times \alpha
$$

where $\alpha$ is the weight factor for distance $D_{i, l}$. When $\alpha$ is greater than 1 , the cluster region of "Not activated" and "Low" becomes smaller and more ovalness. Figure 6 shows the clustering of data from a subject for all three tasks before and after $\alpha$ modification. After multiple trials, 2 and 1.5 are chosen as the optimal value for two and three muscle activation levels tasks, respectively.

Generally, this algorithm outperforms other clustering methods such as K-means orK-medoids clustering algorithmsbecause it is designed for small datasets where the cluster centroids are fixed, making them immune to outliers and noises, which can have noticeable effects on data clustering. The ability to incorporate a scaling factor makes the algorithm conform to the characteristic of muscle activation signals and thus increases clustering efficiency.

\section{Results}

To validate the accuracy of the security system in regards of repeatability, five subjects were asked to test a 4-digits numerical password system where the subjects will have to repeat 10 password inputs into the system according to the predefined values without prior training. The password digit remains the same across all three tasks. However, strength variations for the password are arranged so that each muscle activation level will received equal portions of button pushes. After the testing data is collected from the system, it is classified and compared with the predefined label. 


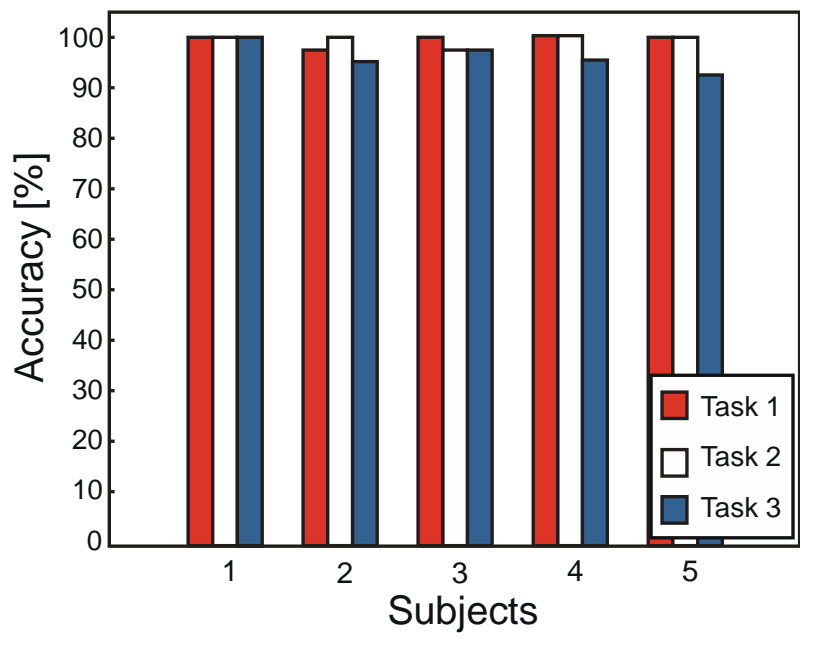

Figure 5. Classification Accuracy of each Subject

Figure 5 illustrates the accuracy for all three tasks. It can be observed that in most cases, tasks with two activation levels can be classified with $100 \backslash \%$ accuracy. This finding can be inferred from the characteristic of the standard deviation for muscle activation levels in Figure 6(a) and (b) which show clear distinction between "Not activated" and "Activated". The result from the third task shows that when there are three or more muscle activation levels, greater control precision is required by the subject in order to make clear difference between neighboring muscle activation levels. This can be illustrated by the overlapping of feature values across different activation levels as shown in Figure 6(c).

\section{Discussions}

With the system fully integrated, the potential to increase security integrity is shown in the Table 1 where criteria such as possible combinations in various cases are listed together with the comparison cases for ATMs and mobile devices. It is shown that, with two and three muscle activation levels, the number of possible input key combinations is increased from 10,000 to 160,000 and 810,000 respectively. This amounts to the increased of time when the password is attacked using automated algorithms such as Brute-Force attack. Even though in the case where a password is leaked, the attacker has to try at most 16 and 81 password combinations, respectively.

This could be proved to be very useful for real-world systems such as ATM machines because the card will be confiscated after three wrong attempts. This means $81.251 \%$ and $96.31 \%$ in failing an ATM attack. For mobile devices such as iPhones where wrong attempts are allowed up to 10 times, the rate falls to $31.50 \backslash \%$ and $78.661 \%$, respectively. Another indirect benefit is that it allows users to have more time to realize the lost of their belongings and call their providers to freeze their credit cards or wipe out important information on their mobile devices. 
(a)

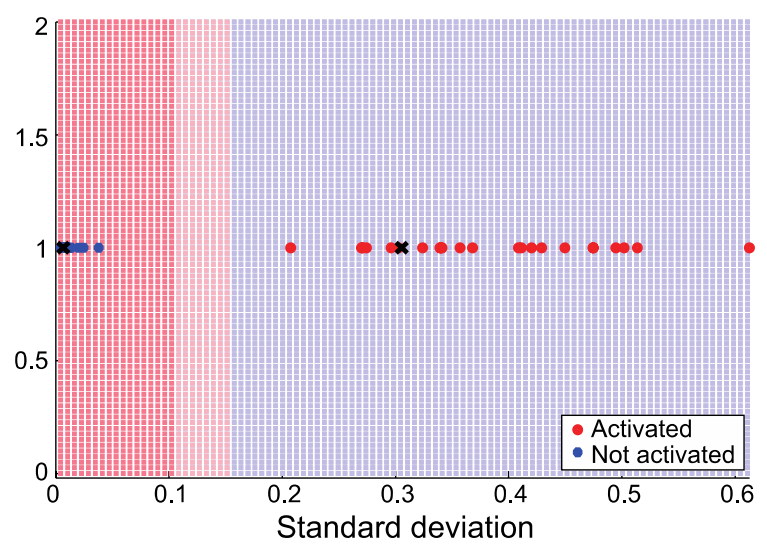

(b)

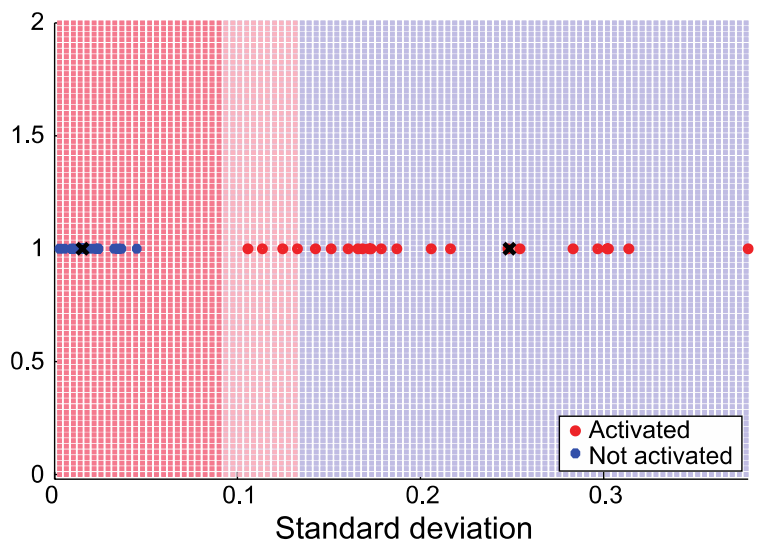

(c)

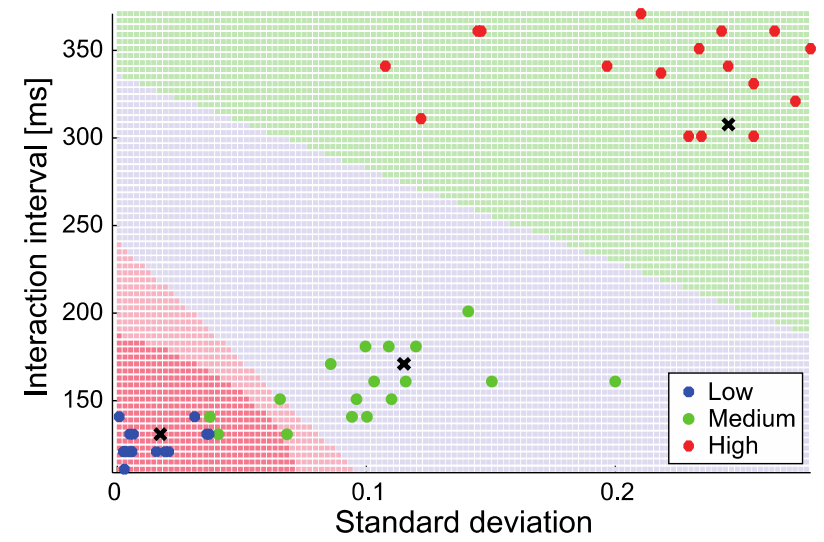

Figure 6. Data Distributions: Clustering Result for the First (a), the Second (b) and the Third (c) Task. The Softer Red Color Depicts the Area for "Low" or "No

Activation" without $\alpha$, whereas the Darker Red Depicts the Area after $\alpha$ is applied. The Values of $\alpha$ used for each Task are 2, 2, and 1.5, Respectively Black Circles Displayed are Cluster Centroids 
Table 1. Potential Increased in Security Integrity for the Proposed System

\begin{tabular}{lcc}
\hline \hline & 2 Levels & 3 Levels \\
\hline Possible entries without password & 160,000 & 810,000 \\
Possible entries with password known & 16 & 81 \\
Percentage of successful ATM attacks & 18.75 & 3.70 \\
Percentage of successful mobile device attacks & 62.50 & 12.34 \\
\hline
\end{tabular}

In the future, additional EMG channels will also be examined for additional distinctive features, such as joint angle, joint torque, or stiffness, which may result in either elimination of time interval consideration or more distinguishable muscle activation levels. Also, in order to handle subject variation problems, additional data will be collected to perform further experiments using non-linear SVM classifier that has greater generalization capability.

\section{Conclusion}

This paper presents an advanced security system by sensing muscle activation from Electromyography (EMG) sensor. In order to combine password characters and muscle activation levels into a password, an algorithm for recognizing different muscle activation levels has been designed. The experimental results have shown that the algorithm performs well for most subjects without any prior learning or training. Also, the usage of the input combination can increase a system's safety without having to modify the original password's length or character variation. The technique is also not limited to only 4-digits numerical passwords. Other password systems with greater input range, such as alphanumeric passwords, can utilize the technique to further increase password complexity. Moreover, the system allows tight integration with any password system without changing the way a password is input into the system.

In conclusion, EMG signals are shown to have the capability of increasing computer security. The muscle activation levels can be detected easily without complex transformations, resulting in ease of implementation. The proposed system can mitigate possible damage from both computer attacks and a theft by prolonging the attacking time or acts as a fail-safe when a user's password is leaked to a third party. With EMG technologies becoming more stable and advanced, this experiment aims to urge the development of a more portable and economic type of EMG sensors that can be used in large scales.

\section{Acknowledgements}

This work was supported by JST CREST (JY240010).

\section{References}

[1] S. Marechal, “Advances in password cracking”, Journal in Computer Virology, vol. 4, (2008), pp. 73-81.

[2] H. Kleinhans, J. Butts and S. Shenoi, "Password cracking using sonyplaystations", in Advances in Digital Forensics V, ser. IFIP Advances in Information and Communication Technology, Edited G. Peterson and S. Shenoi, Springer Boston, vol. 306, (2009), pp. 215-227.

[3] S. Rass, D. Schuller and C. Kollmitzer, "Entropy of graphical passwords: Towards aninformation-theoretic analysis of face-recognition based authentication", in Communications and Multimedia Security, ser. Lecture Notes in Computer Science, Edited B. De Deckerand I. Schaumller-Bichl, Springer Berlin, vol. 6109, (2010), pp. 166-177. 
[4] X. Tan and B. Bhanu, "Fingerprint verification using genetic algorithms", Proceedings of 6th IEEE Workshopon Applications of Computer Vision, (2002) December 3-4, pp. 79-83.

[5] R. Hill, "Retina identification", in Biometrics, Edited A. K. Jain, R. Bolle and S. Pankanti, Springer US, (2002), pp. 123-141.

[6] D. Shin, A. Katayama, K. KIM, J. Kim, N. Yoshimura, H. Kambara and Y. Koike, "A Virtual Instrument System Operated by Electromyographic (EMG) Signals", Information-Tokyo, vol. 16, no. 5, (2013), pp. 3275-3285.

[7] D. Shin, H. Kambara, N. Yoshimura, Y. Kang and Y. Koike, "Control of a Brick-Breaking Game Using Electromyogram", International Journal of Engineering andTechnology, vol. 6, (2014), pp. 128-131.

[8] N. Suresh, X. Li, P. Zhou and W. Z. Rymer, "Examination of motor unit controlproperties in stroke survivors using surface emg decomposition: A preliminary report", Proceedings of the IEEE Engineering in Medicine and Biology Society, (2011) September, pp. 8243-8246.

[9] C. J. De Luca, J. J. Buccafusco, S. H. Roy, G. De Luca and S. H. Nawab, "The electromyographic signal as a presymptomatic indicator of organophosphates in thebody", Muscle nerve, vol. 33, no. 3, (2006), pp. 369-376.

[10] H. Manabe and Z. Zhang, "Multi-stream hmm for emg-based speech recognition", Proceedings of the 26th IEEE Engineering in Medicine and Biology Society, vol. 2, (2004) September, pp. 4389 -4392.

[11] D. Kay, F. E. Marino, J. Cannon, A. St Clair Gibson, M. I. Lambert and T. D. Noakes, "Evidence for neuromuscular fatigue during high-intensity cycling in warm,humid conditions", European Journal of Applied Physiology, vol. 84, (2001), pp. 115-121.

[12] R. Vinjamuri, Z. H. Mao, R. Sclabassi and M. Sun, "A novel architecture for the designof prosthetic and robotic hands", Proceedings ofthe IEEE 32nd Annual NortheastBioengineering Conference, (2006) April, pp. 163-164.

[13] J. S. Kim, H. Jeong and W. Son, “A new means of HCI: Emg-mouse”, Proceedings of IEEE International Conference on Systems, Man and Cybernetics, vol. 1, (2004) October, pp. 100-104.

[14] K. Zintus-art, S. Saetia, V. Pongparnich and S. Thiemjarus, "Dogsperate Escape: A Demonstration of RealTime BSN-Based Game Control with e-AR Sensor," in Knowledge,Information, and Creativity Support Systems, ser. Lecture Notes in Computer Science, Springer Berlin, vol. 6746, (2011), pp. 253-262.

[15] D. Shin, J. Kim and Y. Koike, "A Myokinetic Arm Model for Estimating Joint Torqueand Stiffness From EMG Signals During Maintained Posture”, Journal of Neurophysiology, vol. 101, no. 1, (2009), pp. 387-401.

[16] Y. Koike and M. Kawato, "Estimation of dynamic joint torques and trajectory formation from surface electromyography signals using a neural network model”, BiologicalCybernetics, vol. 73, (1995), pp. 291-300.

[17] D. Shin, H. Watanabe, H. Kambara, A. Nambu, T. Isa, Y. Nishimura and Y. Koike,"Prediction of Muscle Activities from Electrocorticograms in Primary Motor Cortexof Primates”, PLoS One, vol. 7, no. 10, (2012), pp. e47992.

\section{Authors}

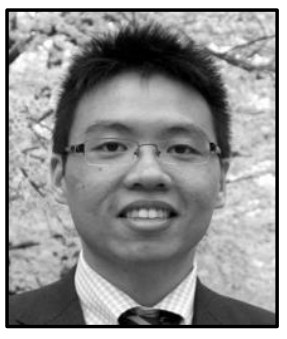

Kalanyu Zintus-art, he received a B. Sc degree in Computer Science from Sirindhorn International Institute of Technology, Thammasat University, Thailand, in 2011. He received aM.Eng in Information Processing from Tokyo Institute of Technology, Tokyo, Japan. His research interests include nonlinear control, adaptive control, human computer interaction, and system identification.

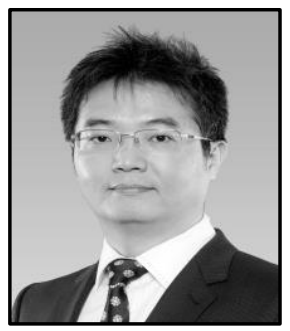

Duk Shin, he received the B.S. and M.S. degrees in engineering in 1996 and 1998 from Chosun University in Korea. He received Ph.D. degree in engineering in 2005 from Tokyo Institute of Technology, Tokyo, Japan. He is now a Project Associate Professor at the Precision and Intelligence Laboratory of Tokyo Institute of Technology.His research interests include brain machine interface (BMI), brain computer interface (BCI), and bio-signal engineering. 


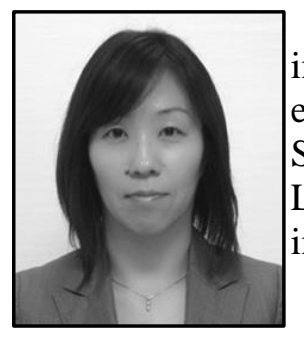

Natsue Yoshimura, she received her M.S degree in medical science in 2006 from Tokyo Medical and Dental University, and Ph.D. degrees in engineering in 2009 from The University of Electro- Communications. She is currently an Assistant Prof. at the Precision and Intelligence Laboratory of Tokyo Institute of Technology. Her research interests include brain machine interface.

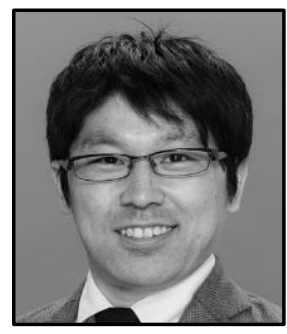

Hiroyuki Kambara, he received the B.Sc. degree in computer science in 2002, M.Sc. and Ph.D. degrees in engineering, in 2004 and 2007, all from Tokyo Institute of Technology, Tokyo, Japan. He is now an Assistant Prof. at the Precision and Intelligence Laboratory of Tokyo Institute of Technology. His research interests include neural motor control and motor learning.

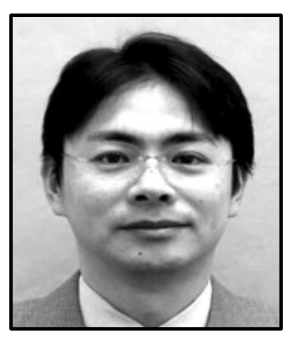

Yasuharu Koike, he received the B.S. and M.S. degrees in computer science in 1987, 1989 and also received Dr. Eng. degree in engineering in 1996, all from Tokyo Institute of Technology, Tokyo, Japan. He worked at Toyota Motors Corporation and Advanced Tele-communications Research (ATR) from 1989 to 1998. He is currently a Professor of Solution Science Research Laboratory, Tokyo Institute of Technology. His research interests include $\mathrm{BMI} / \mathrm{BCI}$ and computational motor learning. 
International Journal of Security and Its Applications Vol.8, No.4 (2014) 